\title{
Mind the gap: how vulnerable patients fall through the cracks of cancer quality metrics
}

\author{
Christopher Manz, ${ }^{1,2,3}$ Katharine Rendle, ${ }^{2,3,4}$ Justin Bekelman ${ }^{2,3,5}$
}

'Department of Medicine, Division of Hematology Oncology, University of Pennsylvania, Philadelphia, Pennsylvania, USA

${ }^{2}$ Penn Center for Cancer Care Innovation, Abramson Cancer Center, University of Pennsylvania, Philadelphia, Pennsylvania, USA

${ }^{3}$ Leonard Davis Institute for Health Economics, University of Pennsylvania, Philadelphia, Pennsylvania, USA

${ }^{4}$ Department of Family Medicine and Community Health, University of Pennsylvania, Philadelphia, Pennsylvania, USA ${ }^{5}$ Departments of Radiation Oncology and Medical Ethics and Health Policy, University of Pennsylvania, Philadelphia, Pennsylvania, USA

\section{Correspondence to}

Dr Christopher Manz, University of Pennsylvania, Philadelphia, PA 19104, USA; Christopher.Manz@ pennmedicine.upenn.edu

Accepted 14 August 2019 Published Online First 23 August 2019

\section{Sinked}

- http://dx.doi.org/10.1136/ bmjqs-2019-009742

\section{Check for updates}

(C) Author(s) (or their employer(s)) 2020. No commercial re-use. See rights and permissions. Published by BMJ.

To cite: Manz C, Rendle K, Bekelman J. BMJ Qual Saf 2020;29:91-94.
In USA, cancer outcomes have steadily improved but considerable disparities in outcomes persist. ${ }^{1}$ There is continued evidence that vulnerable patients (ie, those who are socially or economically disadvantaged) are less likely to receive high-quality care and subsequently have poorer outcomes. ${ }^{2}$ Since the release of the Institute of Medicine's report Ensuring Quality Cancer Care in 1999, increased attention has been paid to the importance of measuring cancer care quality, understanding its effects on outcomes and identifying effective strategies for ensuring that all patients have access to highquality cancer care. ${ }^{3}$ Studies have demonstrated that patient survival varies by hospital type (eg, community vs academic cancer centre), even after risk adjustment for tumour characteristics and comorbidities, and that patients treated at hospitals that perform worse on some cancer quality metrics have inferior survival. ${ }^{4-10}$ Collectively, these findings suggest that variations in cancer care quality translate into decreased survival for thousands of patients every year, and vulnerable patients are at particular risk of poorer cancer outcomes.

The intended goals of quality metrics are to allow hospitals to identify and improve on substandard care, thereby elevating individual and population level cancer care quality, while also enabling patients and payers to choose high-performing hospitals through public reporting. There has been close consideration of how best to measure quality that addresses social drivers of poor cancer outcomes, without punishing hospitals that treat large numbers of vulnerable patients. While quality metrics that focus on the outcomes that matter most to cancer patients-living longer (overall survival) and living better (quality of life)—would be preferred, this is very difficult in practice as these outcomes may indirectly hold hospitals accountable for extrinsic socioeconomic factors beyond their direct control. ${ }^{1112}$

The limitations attached to using survival and quality of life outcomes as quality metrics mean that the substantial majority of cancer care quality measures are process metrics. These quality metrics can, but do not always, correlate with patient survival. ${ }^{6-10} 1314$ Process metrics are often seemingly straightforward to measure, such as whether or not patients for whom guidelines recommend chemotherapy actually receive it, and many have high face validity for being linked to outcomes. As measures of care delivery, hospitals also can more easily improve on poor performance. But process metrics may have well-intentioned design specifications that leave a gap where the most vulnerable patients fall through, meaning that these vulnerable patients may end up receiving worse care.

In this issue of BMJ Quality and Safety, Ryan et al examined the American College of Surgeons Commission on Cancer $(\mathrm{CoC})$ metrics that measure receipt of chemotherapy. ${ }^{15}$ For specific patients with breast, colon and lung cancers for whom US national guidelines recommend chemotherapy, the quality metrics calculate the percentage of eligible patients at an individual hospital who receive recommended chemotherapy. In the current metrics, patients who receive chemotherapy count towards the metric, but hospitals also receive credit for documentation of patients who do not receive chemotherapy because they are too ill or frail, die before administration of planned chemotherapy or are recommended to receive chemotherapy by a clinician but do not receive treatment. This latter 
group of patients for whom clinician-recommended chemotherapy was never administered may represent patients who refuse treatment or receive treatment at a hospital that is not included in the National Cancer Database. However, it may also include patients who wanted to receive chemotherapy but faced obstacles to care that prevented receipt of treatment, such as insurance barriers. Thus, this undertreated population may be misattributed as receiving appropriate care under the current $\mathrm{CoC}$ quality metric.

In their study, Ryan et al compared the current metrics against the total of patients who were recommended to receive chemotherapy but did not receive it. The authors found that the median hospital-level percentage of patients who did not receive clinicianrecommended chemotherapy was $3.2 \%$ for breast cancer, $6.2 \%$ for colon cancer and $9.1 \%$ for lung cancer. There was significant variability between hospitals. Depending on the disease, between 5.6\% and $13.3 \%$ of hospitals were poor performing outliers even after risk adjustment for patient and disease characteristics, with $21.8 \%$ to $40.2 \%$ of the patients at the worst performing hospitals not receiving clinicianrecommended chemotherapy. Although the absolute percentage differences between the groups were small, the study found that the patients at increased likelihood of not receiving clinician-recommended chemotherapy were of non-Hispanic black race, of older age, had a low income, and were either uninsured or had Medicaid coverage. Uninsured and Medicaid patients were lumped together and compared with insured and Medicare patients together, an atypical methodological choice that may mask more nuanced disparities by insurance status. In sum, the findings suggest a substantial number of patients - up to nearly 1 in 10 patients with lung cancer overall, and even more likely in the case of vulnerable populations-did not receive clinician-recommended chemotherapy although they would have presumably benefited from such treatment.

The findings may seem unsurprising given existing evidence of disparities in cancer care. Decades of evidence have documented disparities in cancer outcomes for patients of lower socioeconomic status, racial minorities and those without insurance. ${ }^{1}{ }^{16}$ These socioeconomic and demographic factors are often shown to be associated with survival, and the mediators are multitude, with many potential obstacles in access to care along the entire care pathway.

One of these obstacles is that patients of low socioeconomic status are less likely to receive treatment for their cancer. ${ }^{17} 18$ A survey of cancer patients who reported not receiving recommended cancer care (not just chemotherapy) cited numerous reasons, including that patients could not afford care (72\%), lacked transportation (54\%), could not get child or adult care (50\%), or time off of work (29\%). ${ }^{19}$ In addition to financial and transportation barriers, personal or cultural barriers regarding chemotherapy may also play a role. ${ }^{2021}$ Vulnerable patients may be more sensitive to all of these barriers, and Ryan et al's study pinpoints one of the places on the road to treatment initiation where particularly vulnerable at-risk patients fall through the cracks, which is after clinicians recommend chemotherapy but before the treatment is actually administered. More research is needed to rigorously investigate specific mechanisms driving the relationship of socioeconomic risk factors and failure to receive recommended therapy, and to inform interventions to improve receipt of recommended treatment.

That high rates of patients not receiving clinicianrecommended chemotherapy persisted after risk adjustment for patient, disease and sociodemographic characteristics suggest that patient factors do not explain all of the variation and that hospitals bear some of the responsibility. The construction of the current $\mathrm{CoC}$ metrics absolve hospitals of that responsibility, and perhaps explains why some studies have not consistently shown a correlation between hospital performance on quality metrics and outcomes. ${ }^{71314}$

Ryan et al describe three possible modifications to the $\mathrm{CoC}$ chemotherapy metrics to address this limitation: (1) Adjusting the metrics for socioeconomic risk factors. (2) Keeping the exclusion for failure to receive clinician-recommended treatment but capping the percentage of patients that can contribute positively to a hospital. (3) Removing the exclusion altogether. Of these, only the third option holds hospitals fully accountable for ensuring that patients receive clinicianrecommended therapy or have a justifiable reason for why they did not (primarily, death before receiving planned treatment, patient refusal or treatment at another site). The CoC metrics on receipt of chemotherapy could be modified to allow hospitals to receive credit for patients who receive a recommendation for chemotherapy but do not receive it due to death, refusal or treatment elsewhere, but not to hospitals for patients who are effectively lost to follow-up. If the metrics are modified to report overall performance as well as stratified performance by sociodemographic factors, as recommended by the National Quality Forum, hospitals with large populations of vulnerable patients could be recognised for strong performance for patients of high-risk sociodemographic factors, even if the hospital's overall performance is inferior to the general population. ${ }^{22}$

Still, hospitals may continue to have difficulty determining whether patients who did not receive recommended treatment actually refused treatment and thus did not return for follow-up or did not receive chemotherapy for other reasons. To that end, patient navigation programmes may help patients, hospitals and payers to achieve high-quality care. Establishing cancer care for patients requires negotiating a complex landscape of providers and procedures for a patient to even end up in an oncologist's office with a diagnosis 
sufficient to receive a treatment recommendation. Patients who receive a clinician recommendation for chemotherapy have at least navigated the system that far and may be easier to retain than patients who are lost prior to this point. Hospitals who lose many chemotherapy-eligible patients prior to treatment administration could establish and strengthen patient navigator programmes to ensure patients traverse the last mile to actual receipt of therapy. ${ }^{23}$ Such services might include social work assistance with transportation or insurance obstacles, assistance coordinating appointments with other specialties such as surgery and radiation oncology, pharmacy guidance for filling increasingly common specialty oral medications, and simple phone calls to make sure that patients who do not follow-up have not simply fallen through the cracks. These efforts would also help hospitals clarify which patients truly are refusing recommended therapy and identify remediable causes, such as racial bias or community distrust.

Only 29 hospitals were outliers in more than one disease, though perhaps outlier hospitals for one disease metric were on the poor performing end of normal for other diseases (the lung cancer metric was also newer and only 302 hospitals were included with that metric, vs 1226 and 1275 hospitals for breast and colon cancer metrics, respectively). In addition to further investigation of hospital characteristics linked to high and low performance for these metrics, additional research examining within-hospital variation may help practices identify why patients with specific cancers tend to fall through the cracks and identify strategies to mitigate that loss.

Of course, these services are not free. Understanding that such steps are a necessary part of quality cancer care, payers should reimburse hospitals for provision of patient navigation services. ${ }^{24}$ For instance, Medicare's Oncology Care Model provides a per capita monthly fee of $\$ 160$ to cover care coordination activities. Sustainable financing mechanisms for fee-for-service, managed care and alternative payment models are necessary for hospitals, especially smaller practices, to invest in the personnel and infrastructure to provide navigation services. Patient navigation programmes may even reduce hospitalisations and total healthcare spending, making these services a vital part of value-based care. ${ }^{25}$ Payers could use the revised $\mathrm{CoC}$ metrics and patient demographics to direct additional care coordination payments towards the hospitals with large percentages of vulnerable patients and the greatest coordination needs.

Finally, it is also important to recognise that receipt of treatment is not the same as receipt of optimal treatment. Vulnerable patients are less likely to receive guideline-adherent care, which is associated with worse survival. ${ }^{26}$ In addition, new technologies (eg, chimerical antigen receptor therapy) and new standards of care (eg, routine genetic testing in metastatic lung cancer) may improve on previous care standards, but are not available or adopted instantaneously at all hospitals; the pace of innovation diffusion and implementation also determines whether patients receive optimal treatment. For instance, despite Food and Drug Administration approval of oral targeted therapies for Epidermal growth factor receptor (EGFR)-mutant lung cancer in 2013, EGFR testing rates in community practices in 2014 and 2015 ranged from $100 \%$ to less than $20 \%$ of appropriate patients, inevitably leading to undertreatment of patients whose mutations went undetected. ${ }^{27}$ Strategies are necessary to ensure equitable access to new technologies and standards such that cancer disparities are not exacerbated.

The purpose of cancer care quality metrics is to enable quality improvement and ensure equitable patient access to cancer care that improves patients' lives. The study by Ryan et al illustrates how patients can fall through the cracks of existing metrics and offers a lesson in improvement for our quality metrics.

Funding The authors have not declared a specific grant for this research from any funding agency in the public, commercial or not-for-profit sectors.

Competing interests None declared.

Patient consent for publication Not required.

Provenance and peer review Commissioned; internally peer reviewed.

Data availability statement No additional data are available.

\section{REFERENCES}

1 American Cancer Society. Cancer facts \& figures 2019. Atlanta: American Cancer Society, 2019.

2 Sineshaw HM, Ng K, Flanders WD, et al. Factors that contribute to differences in survival of black vs White patients with colorectal cancer. Gastroenterology 2018;154:906-15.

3 Institute of Medicine. Ensuring quality cancer care. Washington, DC: The National Academies Press, 1999.

4 Shulman LN, Palis BE, McCabe R, et al. Survival as a quality metric of cancer care: use of the National cancer data base to assess Hospital performance. J Oncol Pract 2018;14:e59-72.

5 Pfister DG, Rubin DM, Elkin EB, et al. Risk adjusting survival outcomes in hospitals that treat patients with cancer without information on cancer stage. JAMA Oncol 2015;1:1303-10.

6 Odell DD, Feinglass J, Engelhardt K, et al. Evaluation of adherence to the Commission on cancer lung cancer quality measures. J Thorac Cardiovasc Surg 2019;157:1219-35.

7 Mason MC, Chang GJ, Petersen LA, et al. National quality forum colon cancer quality metric performance: how are hospitals measuring up? Ann Surg 2017;266:1013-20.

8 Khanna A, Saarela O, Lawson K, et al. Hospital quality metrics for radical cystectomy: disease specific and correlated to mortality outcomes. J Urol 2019;202:490-7.

9 Lawson KA, Saarela O, Abouassaly R, et al. The impact of quality variations on patients undergoing surgery for renal cell carcinoma: a national cancer database study. Eur Urol 2017;72:379-86.

10 Shulman LN, Browner AE, Palis BE, et al. Compliance with cancer quality measures over time and their association with survival outcomes: the Commission on cancer's experience with the quality measure requiring at least 12 regional lymph nodes to be removed and analyzed with colon cancer resections. Ann Surg Oncol 2019;26:1613-21. 
11 Porter ME. What is value in health care? N Engl J Med 2010;363:2477-81.

12 Institute of Medicine. Committee on improving the quality of cancer care: addressing the challenges of an aging population. In: Levit L, Balogh E, Nass S, et al, eds. Delivering high-quality cancer care: charting a new course for a system in crisis. Washington D.C: National Academies Press (US), 2013.

13 Kantor O, Wang C-H, Yao K. Regional variation in performance for Commission on cancer breast quality measures and impact on overall survival. Ann Surg Oncol 2018;25:3069-75.

14 Nussbaum DP, Blazer DG, Greenup RA, et al. Quality in cancer care: an assessment of the American College of surgeons Commission on cancer quality measures. J Am Coll Surg 2017;225:S125.

15 Ellis RJ, Schlick CJR, Feinglass J, et al. Failure to administer recommended chemotherapy: acceptable variation or cancer care quality blind spot? BMJ Qual Saf 2020;29:103-12.

16 O'Keefe EB, Meltzer JP, Bethea TN. Health disparities and cancer: racial disparities in cancer mortality in the United States, 2000-2010. Front Public Health 2015;3:51

17 Forrest LF, Adams J, Wareham H, et al. Socioeconomic inequalities in lung cancer treatment: systematic review and meta-analysis. PLoS Med 2013;10:e1001376.

18 Aarts MJ, Lemmens VEPP, Louwman MWJ, et al. Socioeconomic status and changing inequalities in colorectal cancer? a review of the associations with risk, treatment and outcome. Eur J Cancer 2010;46:2681-95.
19 Cancer Support Community. Access to care in cancer 2016: barriers and challenges. Washington, D.C: Cancer Support Community, 2016.

20 Guadagnolo BA, Petereit DG, Coleman CN. Cancer care access and outcomes for American Indian populations in the United States: challenges and models for progress. Semin Radiat Oncol 2017;27:143-9.

21 Warren JL, Butler EN, Stevens J, et al. Receipt of chemotherapy among Medicare patients with cancer by type of supplemental insurance. J Clin Oncol 2015;33:312-8.

22 National Quality Forum. Risk adjustment for socioeconomic status or other sociodemographic factors. Washington, D.C: National Quality Forum, 2014.

23 Ramirez A, Perez-Stable E, Penedo F, et al. Reducing time-totreatment in underserved Latinas with breast cancer: the six cities study. Cancer 2014;120:752-60.

24 Osundina FGK, Downer S, American Cancer Society. Patient navigation in cancer care: review of payment models for a sustainable future, 2019

25 Rocque GB, Pisu M, Jackson BE, et al. Resource use and Medicare costs during lay navigation for geriatric patients with cancer. JAMA Oncol 2017;3:817-25.

26 Pfaendler KS, Chang J, Ziogas A, et al. Disparities in adherence to national comprehensive cancer network treatment guidelines and survival for stage IB-IIA cervical cancer in California. Obstet Gynecol 2018;131:899-908.

27 Ruggiero JE, Rughani J, Neiman J, et al. Real-World concordance of clinical practice with ASCO and NCCN guidelines for EGFR/ALK testing in aNSCLC. J Clin Oncol 2017;35. 